\title{
Das Profil von Kitros in Nord-Griechenland als Typusprofil einer mediterranen Lößprovinz
}

\author{
Von Karl Brunnacker, Köln; Hans-Jürgen Altemüller, Braunschweig \\ und Hans-Jürgen Beug, Stuttgart-Hohenheim \\ Mit 14 Abbildungen und 3 Tabellen
}

\begin{abstract}
$\mathrm{Z}$ us a m m e n $\mathrm{f}$ a s s u $\mathrm{ng}$. In einem kleinen Lager im Tiefland bei Kitros (Makedonien) werden fluviale Bildungen durch kolluviale Sedimente und einige Meter Löß mit fossilen Böden überdeckt. Trotz der makro- und mikromorphologisch sehr ausgeprägten Böden werden die Deckschichten gemäß der allgemeinen Geländesituation, wie der Details im Profilaufbau in die Würmeiszeit gestellt.

Die vier fossilen Böden sind weitgehend an Kalk verarmt, sie sind sehr tonreich und zeigen ausgesprochen verdichtetes Gefüge. Die typologische Ansprache als „rote Mediterranböden“ kann vorerst allerdings nur eine Arbeitsbezeichnung sein. Der tiefste dieser Böden wird dem Zeitabschnitt Amersfoort- bis Broerup-Interstadial, der oberste dem Stillfried B zugeordnet.

Gleichartige Vorkommen wurden außerdem bei Xanthi und in der nördlichen Türkei gefunden. Damit zeichnet sich eine eigenständige Löß- und Paläobodenprovinz ab. Feuchtere bzw. wechselfeuchtere Phasen werden darin durch die Böden angezeigt. Diese sind einem Ablauf zwischengeschaltet, der trockener war, der insbesondere - wie auch sonst - gegen das Hochglazial hin durch aridere Bedingungen bei erheblich abgesenkten Temperaturen ausgezeichnet war.
\end{abstract}

$\mathrm{S} \mathrm{u} \mathrm{m} \mathrm{m} \mathrm{a} \mathrm{ry.} \mathrm{In} \mathrm{a} \mathrm{small} \mathrm{deposit} \mathrm{in} \mathrm{the} \mathrm{lowlands} \mathrm{near} \mathrm{Kitros} \mathrm{(Makedonia),} \mathrm{fluvial} \mathrm{formations}$ are covered by collovial sediments and several meters of loess with fossil soils. In spite of the macro- and micro-morphologically weathered soils, the surface layers conform to the general terrain, as the details of the Würm Iceage show.

The four fossil soils are largely lime deficient, they are very rich in clays and show a markedly condensed texture. The typological classification of "red Mediteranean soil" can, nevertheless, only be an initial specification. The deepest of these soil layers is placed in the period between the Amersfoort and Broerup-Interstadial Ice-ages, the upper most in the Stillfried B.

Similar occurrences were discovered at Xanthi and in the north of Turkey, thereby defining a localized loess and paleo-soil region. More moist, respectively alternatingly more moist phases are indicated by the soils. These date from times falling within a generally drier era which, in particular, as is usual, was distinguished as being nye to high glacial by more arid conditions at low temperatures.

\section{Einleitung}

Nach der bisherigen Kenntnis liegen in SE-Europa die südlichsten Lösse bei Titograd und an der Neretva (Brunnacker, Basler, Ložek, Beug \& Altemüller 1969), im Vardar-Tal (Marcović-Marjanović 1964) sowie als „fluviatile lößartige Sedimente“ in Südbulgarien (Fotakiewa \& Mınkow 1966). Planmäßige Suche nach noch weiter südlich gelegenen Vorkommen hat vor einigen Jahren zum Auffinden des Lößlagers bei Kitros, zwischen Aliakmon und Olymp, geführt. Neuerdings wurden außerdem in N-Griechenland und in der nördlichen Türkei weitere entsprechende Vorkommen entdeckt.

Die Deutsche Forschungsgemeinschaft hat durch Gewährung von Reisebeihilfen die Geländeuntersuchungen in den Jahren 1966 und 1968 ermöglicht. Meine früheren Mitarbeiter Herr Dr. R. Streit und Frau H. Staendeke, haben die röntgenographischen Untersuchungen bzw. Sedimentanalysen durchgeführt. Ihnen, wie allen, die auf sonstige Weise mitgeholfen haben die Untersuchungen zu fördern, danke ich.

K. Brunnacker.

\section{Profil Kitros (K. Brunnacker)}

Der Aufschluß liegt nächst der Hauptstraße von Thessaloniki nach Larissa, nördlich der Ortschaft Kitros.

a. Profila u f b a

Am Nordhang eines flachen Tälchens ( $\mathrm{rd} .30 \mathrm{~m} \mathrm{NN}$ ), in welches das heutige Bachbett etwa $7 \mathrm{~m}$ eingeschnitten ist, sind Gehängesedimente und Löß mit zwischengeschalteten 
Böden aufgeschlossen, die sich vom Unterhang gegen den Hangfuß in vier Horizonte auffächern. Am Hangfuß sitzen diese Deckschichten fluviatilen Bildungen auf (Abb. 1):

Nr. Tiefe in $\mathrm{cm}$

0- 25 Humushaltiger, braungrauer, feinsandiger, schluffiger Lehm, krümelig, kalkhaltig

a $\quad-100$ Hellgraubrauner, lehmiger Schluff, Wurzelkanälchen (zahlreich) und Nadelstichporen randlich kalkverkittet, darin dünne Rostauskleidungen, kalkhaltig, viel Kalkpseudomyzel (7,5 YR 5,5/4)

b $\quad-175$ hellbrauner, lehmiger Schluff, schwach prismatisch-brockig, Nadelstichporen mit Kalk umkrustet, schwach kalkhaltig, wenig Kalkpseudomyzel (7,5 YR 4,5/4)

braunroter, lehmiger Ton, säulig absondernd, prismatisch-brockig, Mn-Flecken auf Bodenkörpern, schwach kalkhaltig, Kalkpseudomyzel, butzenartige Kalkabscheidungen (z. T. Spalten ausfüllend), an der Basis Lößkindel (bis $5 \mathrm{~cm} \phi)(2,5$ YR 4/4)

d $\quad-275$ hellbrauner, stark lehmiger Schluff, grobsäulig absondernd, entlang den Klüften schwache rötlichbraune Tontapeten mit z. T. dendritenartigen Mn- und Rostflecken, Nadelstichporen und dünne Wurzelkanäle, schwach kalkhaltig (5 YR 4,5/4)

braunroter, nach unten brauner, lehmiger Ton, prismatisch-brockig, stark Mn-fleckig, schwach kalkhaltig, an der Basis Lößkindel $(1 \mathrm{~cm} \phi)$ z. T. miteinander versintert (5 YR 4/3)

f $\quad-365$ hellgraubrauner, sandiger, stark lehmiger Schluff, nach unten in sandigen Lehm übergehend, schwach prismatisch-brockig absondernd, auf Bodenkörpern Mn- und Rostflecken, Nadelstichporen und Wurzelkanälchen mit Mn-Belag ausgekleidet, stark kalkhaltig, Kalkpseudomyzel (10 YR 5/4)

-415 schwach humushaltiger, brauner bis rotbrauner, toniger Lehm, Wurzelkanäle mit Kalk umkrustet und mit Rostausscheidungen ausgekleidet, schwach kalkhaltig, viel Kalkpseudomyzel (7,5 YR 3/4)

h $\quad-490$ graubrauner Lehm, unten sandig und mit Kleinkieslagen, schwach prismatisch-brokkig, rost- und Mn-fleckig, Wurzelkanäle mit roten Eisentapeten ausgekleidet, kalkhaltig (10 YR 4,5/4)

i $\quad-590$ braunroter, lehmiger Ton, prismatisch-brockig, Mn-Flecken auf Bodenkörpern, unten mit Kalkpseudomyzel (5 YR 3,5/3).

\section{Kitros}

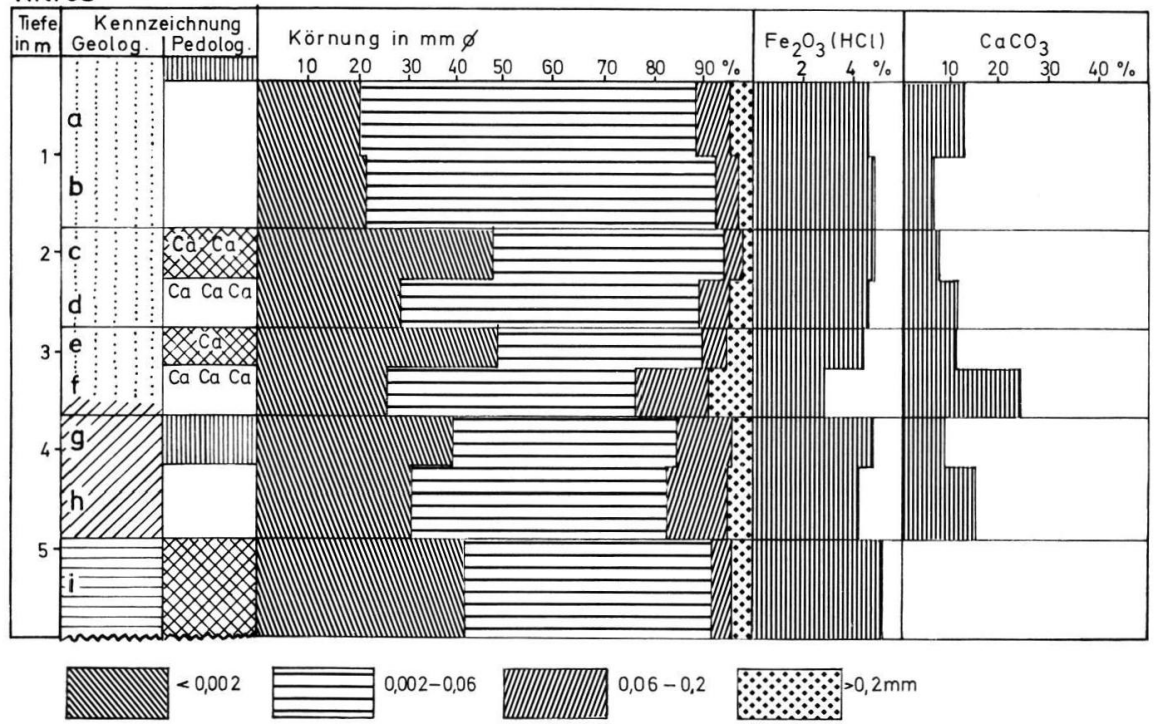

Abb. 1. Profil Kitros. 
Deutung und Versuch einer stratigraphischen Einordnung:

Nr. Tiefe

in $\mathrm{cm}$

$\mathrm{a}+\mathrm{b}$ O_-175 Löß, rezenter Boden weitgehend erodiert

c $\quad-225$ roter Mediterranboden $=$ Stillfried B-Interstadial (PK I)

d -275 Löß

e $\quad-315$ roter Mediterranboden $=$ FWc (vgl. BrunNacker 1957)

f $\quad-365$ Löß über Kolluvium

g -415 Mediterranboden mit Übergang zu Boden mit A-C-Profil = ohne Einordnung

h $\quad-590$ Kolluvium

i $\quad-590$ roter Mediterranboden $=$ Frühwürm bis etwa einschließlich Broerup-Interstadial (PK II)

-590 Talsedimente (R/W-Interglazial?) als mergelige Flußablagerungen mit Kiesschmitzen; am randlichen Hang unter dem Kolluvium wenig verfestigte Mergel (Jungtertiär).

b. A 11 gemeine Befunde

Nachfolgend werden, vor allem unter Verwendung routinemäßiger Laboratoriumsuntersuchungen, die Sedimente und Böden des Profils besprochen.

1. Sedimente

Im tieferen Abschnitt liegen zwischen den Böden und als Ausgangsmaterial derselben fluviatile Bildungen und Kolluvien (Abschnitt $\mathrm{i}-\mathrm{f}$ ), darüber gemäß dem Geländebefund, Löß (Abschnitt $\mathrm{f}-\mathrm{a}$ ). Molluskenreste fehlen. Die Körnung des sehr feinkörnigen Lösses fügt sich im Grundsätzlichen dem bekannten Bild ein (Abb. 2). Die einzige, jedoch nicht entscheidende Abweichung besteht darin, daß die Fraktion 0,002-0,006 $\mathrm{mm} \phi$ etwas höhere Anteile auf Kosten der nächst gröberen aufweist. Dies dürfte entweder mit der speziellen Art des Substrates zusammenhängen, das aus dem Tal des Aliakmon über 20 bis $30 \mathrm{~km}$ Entfernung angeweht wurde, oder es liegen Verschiebungen in der Körnungsverteilung als Folge eines Kalkumsatzes bei und nach Ablagerung des Lösses vor.

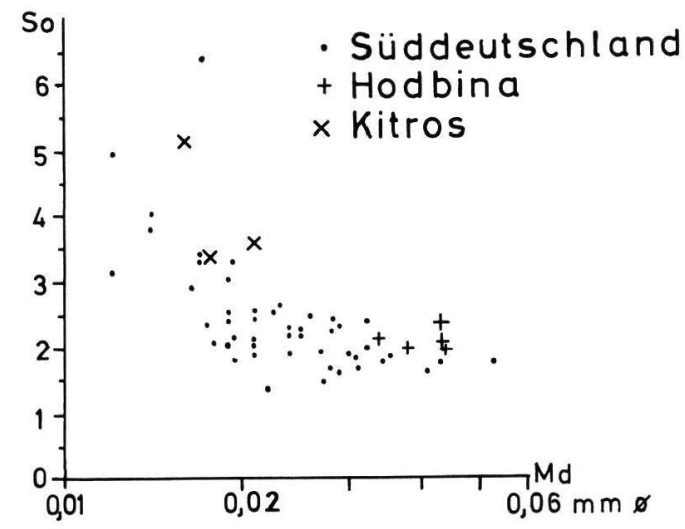

Abb. 2. Medianwert und Sortierungsgrad von Lössen.

\section{Paläoböden}

Der Oberflächenboden ist durch junge Erosion gestört. Im Profil selbst sind vier fossile Böden vorhanden, von denen der unterste (i) der mächtigste ist. Hinsichtlich Färbung, Bodengefüge, Fe- und Mn-Fleckung entsprechen ihm die Böden e und c. Die Ausbildung im Abschnitt g deutet dagegen vielleicht auf eine Übergangsbildung zwischen diesem Typ und einer feuchteren Variante des Tschernosems.

$\mathrm{Fe}$ - und Mn-Ausscheidungen auf den Bodenkörpern geben anscheinend Hinweise auf wechselfeuchte Bedingungen. Rötliche Bodenfarben und scharfkantige Bodengefüge in den Böden i, e und c sprechen ebenfalls für ausgeprägteren Wechsel von stärkerer Durchfeuch- 
tung und kräftigerer Austrocknung, anscheinend bei geringeren Humusgehalten (SCHWERTMANN 1966).

In der röntgenographisch untersuchten Tonfraktion überwiegt insgesamt Montmorillonit (rd. 50-70\%). Illit nimmt in den Lößdeckschichten auf Kosten des Montmorillonits $\mathrm{zu}$ (Abschnitt d bis a). In den Böden (i, g, e und c) erreicht der Illitanteil außerdem etwas höhere Werte als im jeweiligen unterlagernden, frischen Material. Demgegenüber bleibt der geringe Kaolonitgehalt (rd. 5-10\%) im ganzen Profil einigermaßen konstant. Der besonders hohe Montmorillonitgehalt des tieferen Profilabschnittes dürfte mit der Gesteinsausbildung in nächster Umgebung des Profils (Mergel) in Beziehung stehen. Der im oberen Profilteil etwas ansteigende Illitgehalt hängt wohl mit der Lößanwehung aus Gebieten mit etwas abweichender Materialführung zusammen. In den Bodenhorizonten kann er außerdem jeweils mit der Verwitterung in Verbindung gebracht werden.

Die Böden verfügen, trotz anscheinend recht intensiver Verwitterung, über relativ hohe Kalkgehalte. Innerhalb der einzelnen Bodenkörper scheint allerdings weniger Kalk vorhanden als in deren Randzonen. Ferner nimmt der Kalk von der Horizontoberkante nach unten etwas ab. Beide Befunde zeigen auf eine gewisse Kalk-Illuviation in den Böden nach deren Fossilisierung. Daneben ist durchaus denkbar, daß während der Bodenbildung nicht nur Kalk aus dem Boden ausgewaschen wurde, sondern zeitweilig - vielleicht im Zusammenhang mit schwacher "Pseudogley"-Dynamik - darin auch etwas angereichert wurde.

Noch schwieriger ist es, eine Erklärung für die hohen Tongehalte der Böden zu finden. Im tiefern Profilbereich können sedimentationsbedingte relativ hohe Tongehalte das Körnungsbild der Böden von vornherein beeinflussen. Deutlichere Tontapeten entlang den Bodenkörpern fehlen. Das Zurücktreten der Nadelstichporen und Wurzelkanälchen in den Böden spricht hingegen für eine sehr starke Verdichtung des Rückstandes im Verlauf der Pedogenese.

Die Frage des angedeuteten Kalkumsatzes, des hohen Tongehaltes der Böden, wie die Gesamtprägung dieser Paläoböden läßt sich weder über Profilausbildung noch Routineanalysen, sondern am ehesten über mikromorphologische Untersuchungen einer Klärung näher bringen (vgl. Abschnitt c). Damit bleibt aber auch die Bezeichnung als „Roter Mediteranboden " eine vorläufige Benennung.

c. Mikroskopische Dünnschliffuntersuchungen (H.-J. Altemüller)

Aus dem Profil von Kitros wurden Bodenproben mit ungestörtem Gefüge zu Dünnschliffen von etwa $2 \times 3 \mathrm{~cm}$ Größe und $15 \mu \mathrm{m}$ Dicke verarbeitet. Als Einbettungs- und Kittmittel diente das Polyesterharz Vestopal 130 mit einem Brechungsindex $n=1,56$.

Die einzelnen Abschnitte des Profils haben zum Teil ähnliche oder auch übereinstimmende mikroskopische Merkmale. So sind die Lößschichten z. B. nur wenig voneinander verschieden. Auch die tieferliegenden Kolluvien haben noch Merkmale mit dem Löß gemeinsam. Sie unterscheiden sich vor allem durch den größeren Anteil von Sand-Korngrößen, der den Lössen fehlt. Die beiden Bodenhorizonte c) und e) sind vollkommen gleichartig und haben auch eine Ahnlichkeit mit dem an der Basis des Profiles liegenden Boden i). Der schwach entwickelte Boden g) nimmt eine gesonderte Stellung ein.

\section{Befunde}

Die mineralogis che $\mathrm{Z}$ us a m mensetzung der Körner über etwa $10 \mu \mathrm{m} \phi$ ist im gesamten Profil ähnlich. Wesentliche Abweichungen zeigen sich nur in den Bodenhorizonten und sind vermutlich eine Folge der Bodenbildung. Die wichtigsten Mineralarten sind, soweit sie aus dem Dünnschliff mit festem Brechungsindex des Einbettungsmittels bestimmbar waren, nachstehend etwa in der Reihenfolge ihrer Häufigkeit aufgeführt. 
Quarz kommt zahlreich in rundlichen bis splitterartigen Körnern vor, die nicht selten undulöse Auslöschung zeigen. Größere Körner sind zum Teil pflasterartig verwachsen.

A lkalifeldspäte sind vor allem als Orthoklas vorhanden. Charakteristisch ist die deutliche Spaltung und die niedrige Lichtbrechung. Relativ häufig ist auch Mik roklin, der an seiner Gitterlamellierung bei gekreuzten Polarisatoren erkennbar wird.

P la g i ok las e sind leicht zu erkennen, wenn Zwillingslamellen bei gekreuzten Polarisatoren sichtbar werden. Bei Körnern, die aus einzelnen Lamellen entstanden sind, ist Verwechslung mit Quarz möglich. Da die Werte der Lichtbrechung jedoch in vielen Fällen deutlich kleiner als bei Quarz sind (Albit, Oligoklas), kann man an den dünnen Rändern der Schliffe mit Hilfe des Phasenkontrastes die Unterschiede wahrnehmen. Einige Körner wurden auch mit gleichem und etwas höherem Index als Quarz gefunden (Andesin ?). Diese zeigten starke Lösungserscheinungen.

Muscovit ist in allen frischen Schichten des Profils in reichlicher Menge beteiligt, er tritt aber in den Bodenhorizonten stark zurück. Die Plättchen kommen in allen Größen vor und sind zum Teil deutlich aufgespalten oder feinschuppig zerteilt. Es ist nicht auszuschließen, daß sich in dieser Gruppe auch andere Phyllosilikate verbergen.

B i o t it ist ebenfalls zahlreich in den frischen Sedimenten und fehlt in den Bodenhorizonten. Die Kristallplättchen zeigen ausgeprägten Pleochroismus von hell gelblich nach dunkel gelbbraun. Viele sind deutlich angewittert, enthalten Einschlüsse und haben unscharfe, zum Teil aufgeweitete Randzonen.

Chlorit entspricht mengenmäßig etwa dem Biotit und tritt wie die beiden vorgenannten Minerale auf; er fehlt in den Bodenhorizonten. Die Chlorite sind charakteristisch grün gefärbt, haben sehr geringe Doppelbrechung und einen negativen optischen Charakter im Verhältnis zur Länge, in Schnitten senkrecht zur Plättchenebene. Vom Rand her sind sie jedoch alle wie folgt verändert: die Farbe wechselt von grün nach gelblich, die Doppelbrechung nimmt stark zu und der optische Charakter wird positiv zur Länge.

$\mathrm{H}$ or $\mathrm{nblenden}$ sind in kurzsäuligen Formen mit deutlicher Spaltung parallel zur Länge häufig vorhanden. Sie zeigen deutlichen Pleochroismus von nahezu farblos bis grau-grün. Auch eine braune Hornblende kommt nicht selten vor, deren Pleochroismus besonders ausgeprägt ist mit Absorptionen von hell gelblich bis tief rötlich-gelb-braun. Alle Formen, besonders die grünlichen sind stark angewittert und haben auffallende Korrosionsformen mit zackigen Spitzen parallel zur c-Achse.

E p idot tritt in körnigen, bisweilen auch etwas länglichen Formen zahlreich auf. Charakteristisch sind die übernormalen Interferenzfarben, die großen Achsenwinkel und die häufig zu beobachtenden Austritte einer optischen Achse (Lochblende !). In dieser Gruppe ist auch Z o is i t enthalten, der sich in einigen Fällen nachweisen ließ.

T it a n it ist ebenfalls häufig. Es kommen meist körnige Exemplare, aber auch größere Kristalle mit deutlichem Spaltsystem vor, das die bekannten rautenförmigen Querschnitte erkennen läßt. In geringerer Anzahl sind auch feinstkörnige Aggregate anzutreffen (Leukoxen).

C a lcit, D olomit (?) kommen als skelettbildende Korngrößen nur in den frischen Kolluvien in nennenswerten Mengen vor. Es handelt sich meist um Einzelkristalle oder Verwachsungen aus größeren Kristallen, zum Teil mit deutlichen Zwillingslamellen. In einigen Fällen sind zackige Korrosionsformen ausgebildet. Ein Nachweis auf Dolomit wurde nicht geführt.

Weitere Minerale sind:

A patit (angewittert), Topas, Rutil, Zirkon, Disthen, Granat. Außerdem kommen opake Körner (Magnetit ?), braune Körner von Fe-Gel, gelbliche, glaukonitähnliche Aggregate und andere in untergeordneter Menge vor.

Die sekundären Ausscheidungen von Calcit, sowie von Mangan- und Eisenhydroxiden werden in der folgenden $\mathrm{G}$ e f ü ge bes ch r e ibung erwähnt.

$$
\text { Der obere Löß, O-175 cm, Proben a) und b) }
$$

Die Abb. 3 zeigt eine Ubersicht des Gefüges bei schwacher Vergrößerung mit einem lockeren, aber kohärenten Kornverband in welchen röhrenförmige Hohlräume, die sogenannten Nadelstichporen mit Durchmessern von im Mittel 0,2 bis 0,4 mm eingeformt sind. Die umgebenden dunklen Saumbildungen werden durch Calcit-Mikrolithe verursacht, die das Gefüge in den Randzonen durchsetzen und zum Teil verkitten.

Die gröberen Schluffkörner $(20-60 \mu \mathrm{m})$ bilden stellenweise ein sperriges Gefügeskelett oder sind so aneinandergelagert, daß sich verwachsene Aggregatkomplexe abzeichnen.

Die tonigen Anteile sind im Schliff hell gelblichgrau durchscheinend und bilden dünne Beläge um freie Kornoberflächen oder Füllmassen in Kornzwischenräumen. Im unteren Teil des Löß (Probe b) wird die Färbung intensiver braun. Bei gekreuzten Polarisatoren 


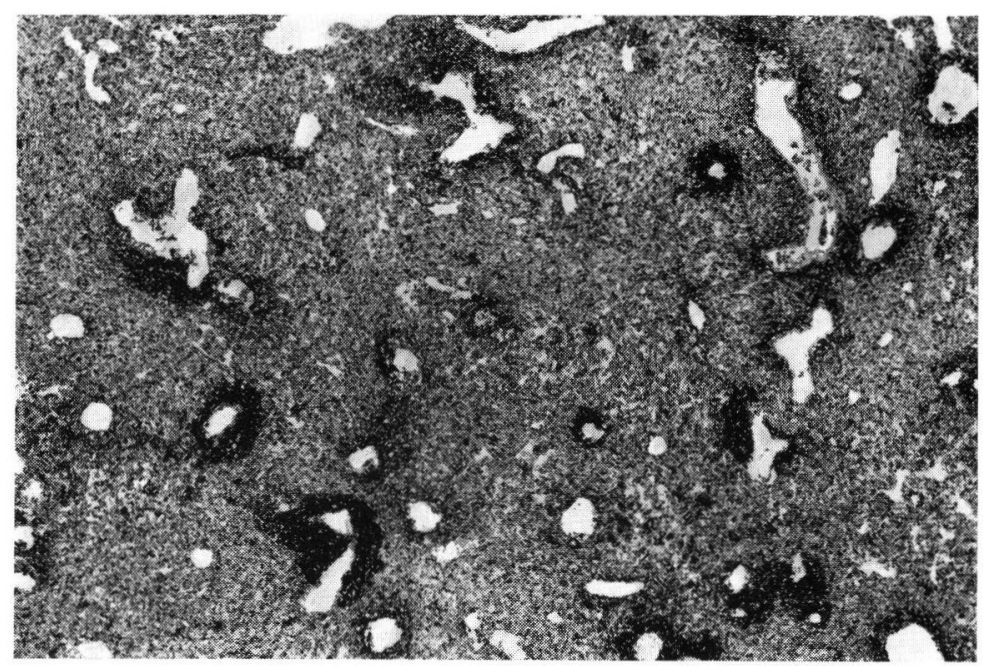

Abb. 3. Ubersichtsbild, oberer Löß, Probe a: Kohärentes Gefüge aus locker gelagerten Grobschluffkörnungen. Zahlreiche röhrenförmige Hohlräume (Nadelstichporen) mit dunklen Randzonen durch Calcit-Ausscheidungen. - Abbildungsmaßstab $\mathrm{M}=8: 1$. - Objektiv Leitz Photar 6,3 : 1, Hellfeld, Durchlicht, Adox Rollfilm R 14.

und mittlerer bis starker Vergrößerung zeigen die tonigen Anteile Richtungsdoppelbrechung in Kornbelägen und fleckige Orientierungen in den Füllmassen.

An den Wänden der Nadelstichporen sind, besonders im oberen Teil des Löß, geschichtete Feintonmassen orientiert angelagert. Ahnliche Beispiele aus einem tieferen Löß sind in den Abb. 7-10 dargestellt. Die Tonbeläge sind fast immer über den Calcit-Ausscheidungen angeordnet und werden nur sehr vereinzelt auch von Calcit überlagert.

Mangan- oder Eisenhydroxidausscheidungen sind im oberen Teil (Probe a) selten, im unteren Teil (Probe b) etwas häufiger vorhanden, doch meist nur als kleine Trümmer von Konkretionen und nur andeutungsweise als Neuausscheidungen.

\section{Der obere Bodenhorizont, $175-225 \mathrm{~cm}$, Probe c)}

Bei hohem Tongehalt ist hier ein dichtes, braunlehmartiges Gefüge mit einer rötlichgelbbraun durchscheinenden Grundmasse ausgebildet, in welche die Feinskelettkörner eingeschlossen sind. Zur Übersicht kann die Abb. 4 herangezogen werden, die zwar den tieferen Bodenhorizont darstellt, der aber dieselben Merkmale aufweist. Man erkennt die glatten Risse, die den Boden ziemlich vollständig in polyedrische Körper zerteilen. Die Feinskelettkörner sind so dicht von der Tonmasse umschlossen, daß sie am frischen, nicht präparierten Boden auch unter der Lupe kaum sichtbar werden. Man glaubt, einen reinen, wachsartig glänzenden Ton vor sich zu haben. Erst im Dünnschliff wird die eingeschlossene Lößkörnung sichtbar.

An den Wandungen der Risse sind streckenweise schwarzbraune, krustenartige Ausscheidungen von Manganhydroxiden vorhanden (Abb. 11 und 12), die man an frischen Bodenproben als schwarze, glänzende Flecken mit unregelmäßiger Umgrenzung beobachten kann (Abb. 13). ${ }^{1}$ )

Stellenweise finden sich im Inneren der tonreichen Grundmasse auch sekundäre CalcitAusscheidungen als Sammelkristallisationen aus Mikrolithen, zum Teil mit Einschluß der Tonmasse. Interessant sind auch die verstreut vorkommenden Calcitausscheidungen an Oberflächen, wie sie zum Beispiel in Abb. 12 mit fast idealen Rhomboederformen aufgenommen sind. 


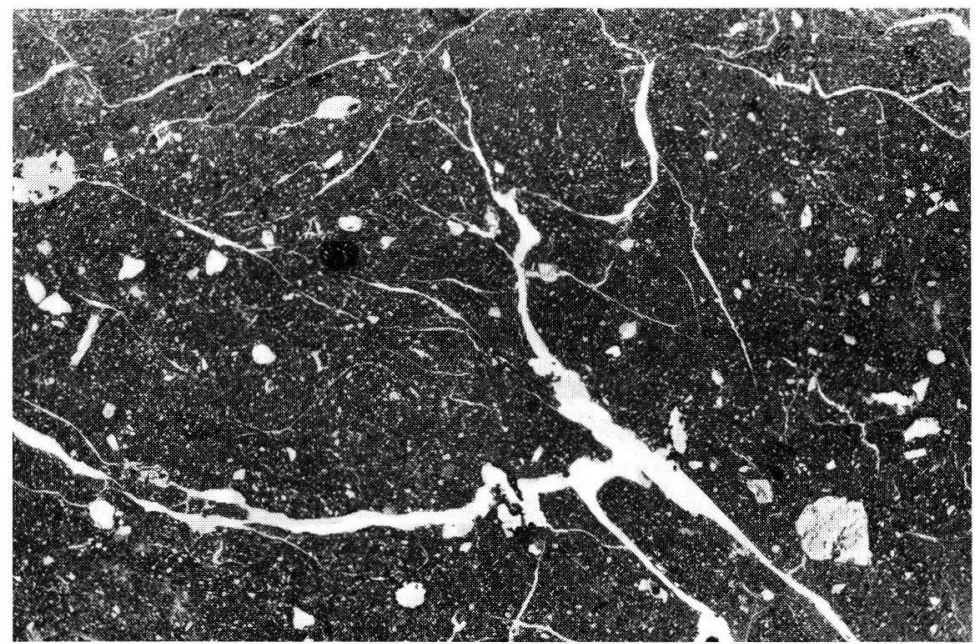

Abb. 4. Übersichtsbild, fossiler Boden, Probe e: Dichtes, braunlehmartiges Gefüge mit glatten Schwundrissen. - Techn. Daten wie Abb. 3.

Irgendwelche Ansammlungen von Feinton mit geschichteten Anlagerungsgefügen, z. B. in Klüften oder in den wenigen röhrenförmigen Hohlräumen, fehlen vollkommen. Auch im Gefügeinneren sind keine Merkmale für eine vorausgegangene Toneinlagerung erkennbar. Die Grundmasse ist im Durchlicht-Hellfeld nahezu homogen und zeigt bei gekreuzten Polarisatoren und mittlerer bis starker Vergrößerung regellos verteilte, ineinander übergehende doppelbrechende Komplexe von einigen $\mu \mathrm{m}$ Größe, die nur an den Rändern der Skelettkörner oder entlang von Spalten als Saumbildungen und Schlieren etwas mehr übergeordnete Orientierungsgrade erreichen.

\section{Löß, $225-275 \mathrm{~cm}$, Probe d)}

Das Gefüge ist dem oberen Löß sehr ähnlich, jedoch ist ein größserer Anteil von braunem, tonigem Material vorhanden, das zum Teil als Bodentrümmer erkennbar und von braunlehmartiger Beschaffenheit ist. Daraus folgt die intensivere Braunfärbung des Löß und das Auftreten der Risse. Außerdem sind noch folgende Merkmale ausgeprägt:

Beläge von geschichtetem Feinton mit guter Richtungsdoppelbrechung und intensiver rötlich-gelbbrauner Färbung sind an den Wänden der zahlreichen Nadelstichporen und anderer Hohlräume angelagert (Abb. 7 bis 10). Auf den Tonbelägen befinden sich häufig krustenartige Ausscheidungen von Manganhydroxiden, die sich teilweise auch schuppenartig in die Tonbeläge hinein fortsetzen.

Der zweite Bodenhorizont, $275-315 \mathrm{~cm}$, Probe e)

Das Gefüge entspricht vollkommen dem zuerst beschriebenen Bodenhorizont. Es ist in Abb. 4 als Übersicht bei schwacher Vergrößerung dargestellt. Manganhydroxid-Krusten und verstreut eingelagerte Calcit-Ausscheidungen sind wie oben vorhanden. Merkmale einer Toneinlagerung fehlen völlig.

$$
\text { L öß über Kolluvium, 315-365 c m, Probe f) }
$$

Hier zeigen sich überwiegend die Merkmale, die schon beim oberen Löß, Probe a), beschrieben wurden. Bei den Skelettmineralen nehmen jedoch größere Körnungen (über $60 \mu \mathrm{m} \phi)$ zu. Typisch sind wieder die Nadelstichporen mit ihren randlichen Niederschlägen aus Calcit-Mikrolithen. Carbonatminerale sind hier auch in größeren Körnern am Skelett beteiligt. Glimmerartige Minerale sind zahlreich vorhanden. 


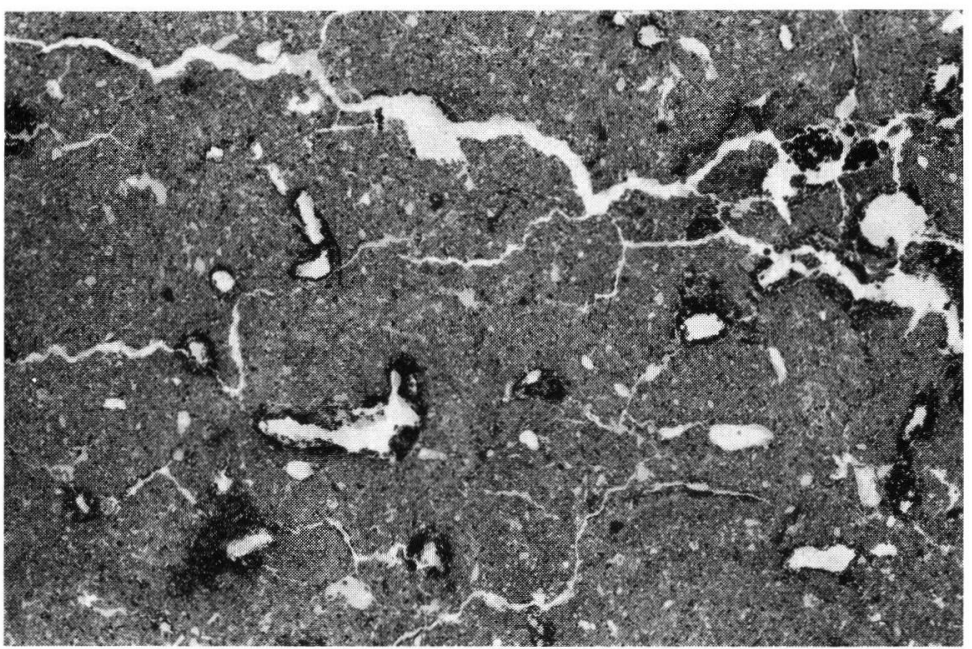

Abb. 5. Übersichtsbild, schwach entwickelter Boden, Probe g: Kohärentes Grundgefüge mit Schwundrissen. Nadelstichporen mit dunklen Säumen von Manganhydroxiden und teilweise Calcit-Ausscheidungen. Techn. Daten wie Abb. 3.

Die tonigen Anteile um die Skelettkörner und in Kornzwischenräumen sind gelblichgrau durchscheinend und bilden schwach doppelbrechende Säume. Von diesen sind die kräftig gefärbten rötlich-gelbbraunen Feintonbeläge in Nadelstichporen, die hier ebenfalls häufig sind, deutlich zu unterscheiden. Sie sind etwa den Belägen in den Abb. 7-10 ähnlich, aber es fehlen meist die Manganhydroxid-Krusten. Mn-Ausscheidungen sind mehr im Inneren des Gefüges als kleine Flecken in Kornzwischenräumen vorhanden.

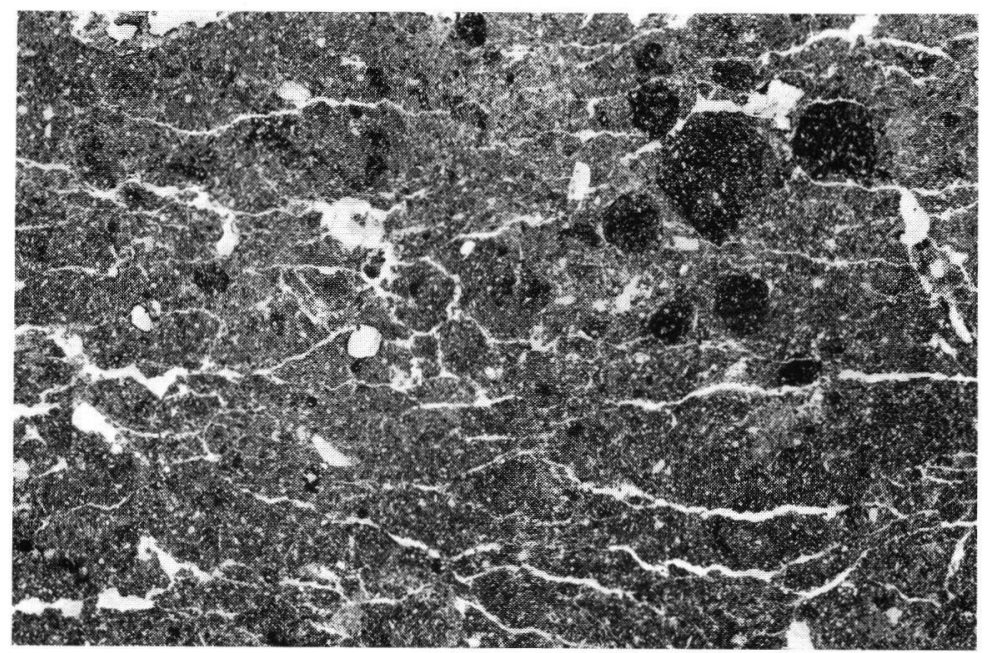

Abb. 6. Übersichtsbild, fossiler Boden, Probe i: Kohärentes, braunlehmartiges Grundgefüge mit angenähert parallel verlaufenden Rissen. Verstreut Fe-Mn-Konkretionen. Techn. Daten wie Abb. 3.

1) An freipräparierten Flecken gelingt der Nachweis des Mangan leicht mit einer SodaSchmelze. 
Schwach entwickelter Boden, 365-415 c m, Probe g)

Dieser Bodenhorizont ist tonreicher als die benachbarten Profilabschnitte und besitzt ein ziemlich dichtes Gefüge. Wie Abb. 5 in der Ubersicht zeigt, ist dieser durch Schwundrisse unregelmäßig gegliedert. Die Färbung ist im Dünnschliff blaß gelblichgrau und nur um weniges intensiver als in dem darunterliegenden Kolluvium. Die zahlreichen Nadelstichporen sind von gleicher Größe wie im Löß (vgl. Abb. 3). Einzelne sind auch von Calcit-Ausscheidungen umgeben. Die Mehrzahl von ihnen ist jedoch von schwarzbraunen Mn-Krusten ausgekleidet. Auch Feinton-Beläge von rötlich-gelblichbrauner Farbe sind in diesen Leitbahnen vorhanden.

Das Innengefüge ist zum größten Teil carbonatfrei. Die Skelettkörner sind in diesen Partien ziemlich dicht gelagert und ringsum von deutlich doppelbrechenden Säumen aus hellem Tonmaterial umgeben. Größere doppelbrechende Schlieren fehlen. Glimmerartige Minerale sind in großer Menge vorhanden, aber meist als kleine Schuppen und Blättchen in die Tonmasse eingearbeitet.

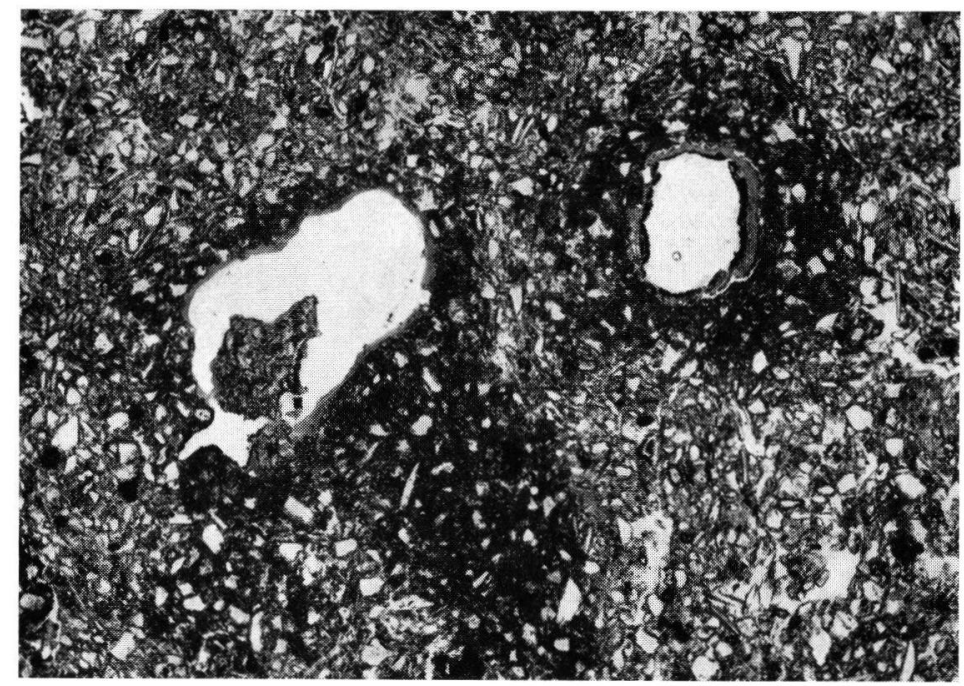

Abb. 7. Löß, Probe d: Nadelstichporen mit dünnen Tonbelägen und Calcit-Ausscheidungen. $\mathrm{M}=40: 1$. - Objektiv Leitz Pl 4/0,10 Hellfeld, Durchlicht, Film Adox KB 14 (alle folgenden Abb. dto.)

$$
\text { Kolluvium, 415-490 c m, Probe h) }
$$

Für dieses Material trifft weitgehend die Beschreibung der Probe f) zu. Ergänzend muß auf einen größeren Anteil von Sand hingewiesen werden, der auch Gesteinstrümmer enthält, die vor allem kristallinen Schiefern angehören. Der Anteil der glimmerartigen Minerale ist wieder hoch, und Carbonate sind sowohl im Skelettanteil als auch in feinkristallinen Formen als Mikrolithe und als nadelförmiger Lublinit gleichmäßig verteilt vorhanden. Zum Teil finden sich auch rundliche Manganhydroxid-Konkretionen und braunlehmartige Bodentrümmer mit oder ohne randliche Mn-Krusten (Abb. 14).

$$
\text { Derunterste Bodenhorizont, 490-590 cm, Probe i) }
$$

Dieser Horizont ist vor allem den beiden braunlehmartigen Bodenhorizonten c) und e) ähnlich. Es ist ebenfalls eine rötlich-gelbbraune, dichte Ton-Grundmasse vorherrschend, in welche die Skelettkörner eingebettet sind. Durch den geringeren Tongehalt ist der Braunlehmcharakter jedoch weniger stark ausgeprägt. Eine Úbersicht über das Gefüge bei schwacher Vergrößerung gibt Abb. 6. 
Die Färbung ist im Dünnschliff infolge einer ungleichen Verteilung tonreicher und tonärmerer Partien und infolge einer Trennung in stärker und schwächer gefärbte Zonen etwas fleckig. Zum Teil erkennt man deutliche Grenzen. Die tonigen Anteile zeigen nur um die Skelettkörner doppelbrechende Säume. Schlieren im Gefügeinneren oder Orientierungen an den Rißoberflächen sind nicht zu beobachten. Ebenso fehlen alle Merkmale für eine Einlagerung von Feinton, wie z. B. geschichtete Beläge in Klüften oder Röhren.

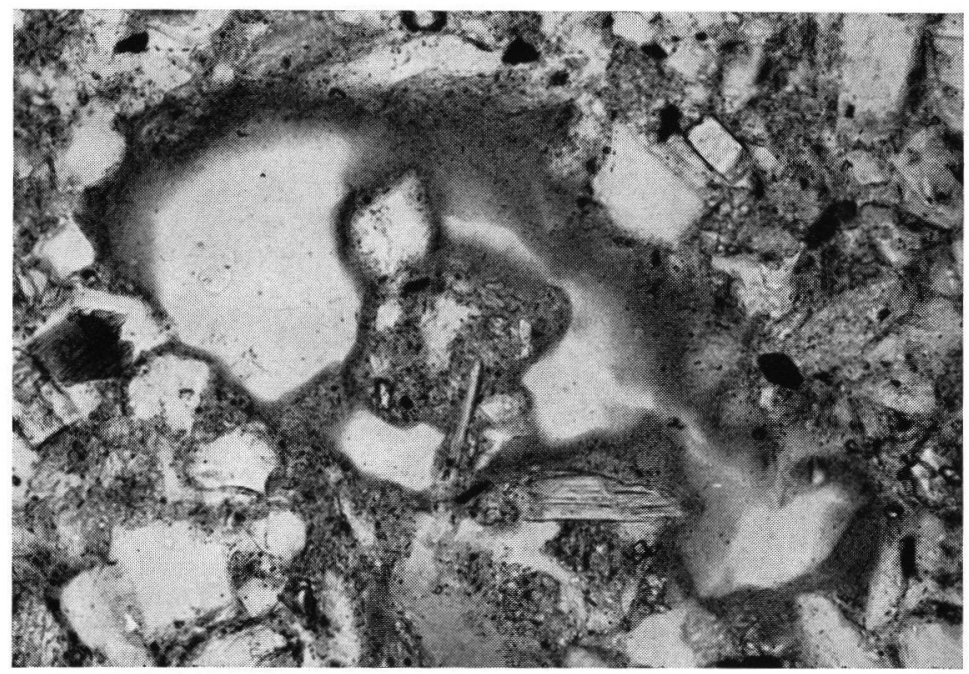

Abb. 8. Löß, Probe d: Dünner Tonbelag in einer Pore über calcitreichem Material. $M=250: 1$. Objektiv Leitz Pl 25/0,50.

2. Besprechung der Ergebnisse

Nach den mikroskopischen Untersuchungen ist der L öß im Profil von Kitros besonders in der jüngsten Schicht, die durch die Probe a) repräsentiert wird, mit typischen Merkmalen ausgebildet. Vergleicht man diesen Löß mit den äolischen Sedimenten des NeretwaTales (Brunnacker u. Mitarbeiter 1969), insbesondere dem Lößprofil von Hodbina, so zeigt sich als wesentlicher Unterschied die völlig andersartige Mineralzusammensetzung der skelettbildenden Korngrößen. Der Löß von Hodbina nimmt durch seinen dominierenden Gehalt an Dolomit eine Sonderstellung ein. In Kitros ist der Gehalt an Ca-Carbonaten im Wesentlichen auf die sekundären mikrolithischen Calcitausscheidungen zurückzuführen. Teile des Innengefüges sind sogar carbonatfrei. Vielleicht hat im ursprünglichen Löß ein größerer Carbonatanteil auch im Gefügeskelett existiert. Aus dem Vergleich mit dem tieferliegenden Kolluvium könnte diese Folgerung abgeleitet werden. Zuverlässige Hinweise gibt es noch nicht.

Der obere Löß nimmt nach der Tiefe einen mehr bräunlichen Farbton an. Auch der Löß zwischen den beiden Bodenhorizonten - Probe d) - ist auffallend braun. Dieses Phänomen der Braunfärbung einer jüngeren Lößdecke über einem begrabenen Bodenhorizont wurde schon in Hodbina beobachtet. Dort wurde vermutet, daß der Löß aufgearbeitetes Bodenmaterial enthält. Dabei bleibt es offen, ob der Boden als äolisches Material transportiert oder am Ort eingearbeitet wird. Durch die intensivere Bodenbildung in den fossilen Böden von Kitros läßt sich dieser Vermischungsprozeß hier mit größerer Sicherheit darstellen. Man erkennt deutlich, daß in die Lößschichten mehr oder weniger gut erhaltene Trümmer von braunlehmartigem Bodenmaterial eingearbeitet sind, die den anschließenden Bodenhorizonten völlig entsprechen. Auch in das Kolluvium über dem untersten Bodenhorizont sind Bodenreste eingearbeitet, es ist aber keine Farbveränderung 
äußerlich zu erkennen. Hier wirkt vielleicht der höhere und gleichmäßiger verteilte Carbonatanteil maskierend. Überdies sind die Bodentrümmer häufig von Manganhydroxiden überkrustet, wie es das Beispiel in Abb. 14 zeigt.

Aus den Dünnschliffbeschreibungen geht ferner hervor, daß im Löß braunlehmartige Feintonbeläge an den Wänden der Nadelstichporen vorkommen. Dieser Ton ist wohl aus den jeweils darüberliegenden Bodenhorizonten eingewaschen. Man kann zwar nicht ganz ausschließen, daß auch örtlich eingemengtes Bodenmaterial zur Belagbildung beigetragen hat, die Tonbeläge finden sich aber auch dort, wo eine solche Bodeneinmischung nicht zu beobachten ist. So kann man z. B. in dem schwach entwickelten Bodenhorizont - Probe g) - keine Merkmalsbeziehungen zwischen dem Ton im Bodeninneren und dem Ton der Beläge herstellen.

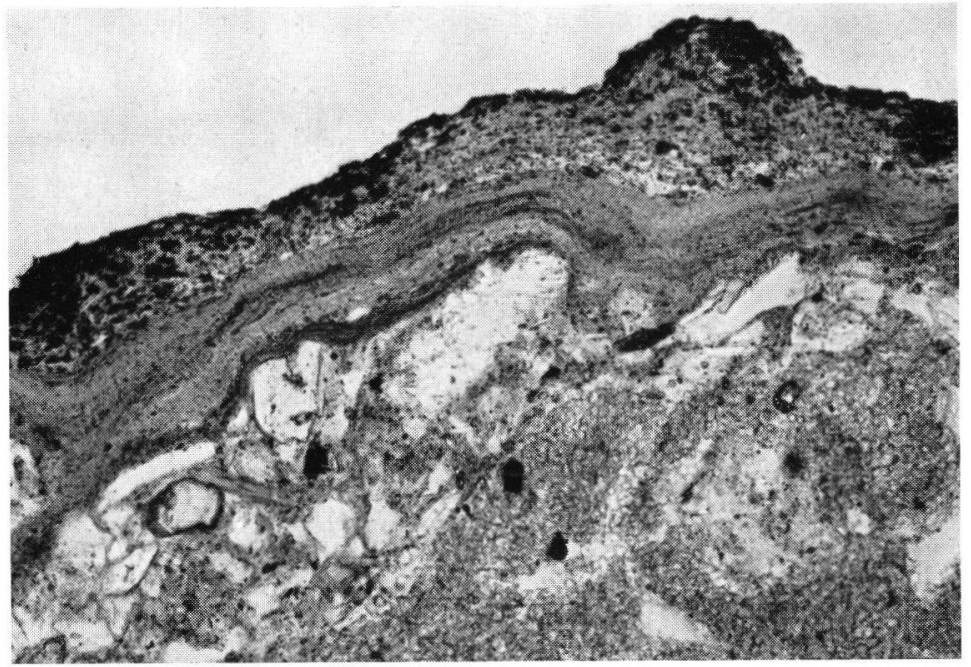

Abb. 9. Löß, Probe d: Deutlich geschichteter Tonbelag mit Kruste aus Mn-Hydroxiden. Darunter calcitreiches Material. - Techn. Daten wie Abb. 8.

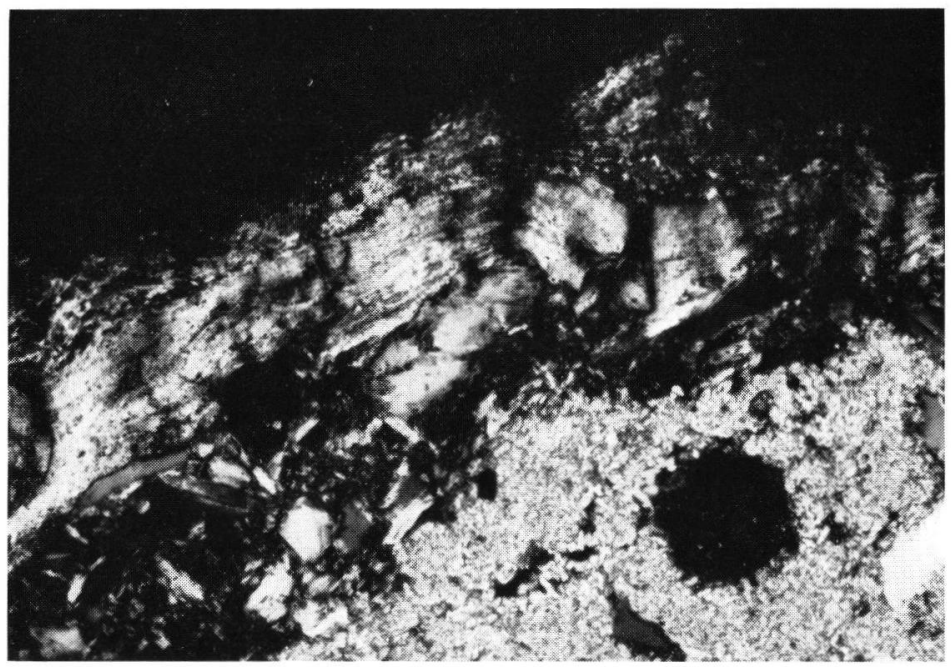

Abb. 10. Gleiches Bild bei gekreuzten Polarisatoren. Deutliche Richtungsdoppelbrechung im Tonbelag. 


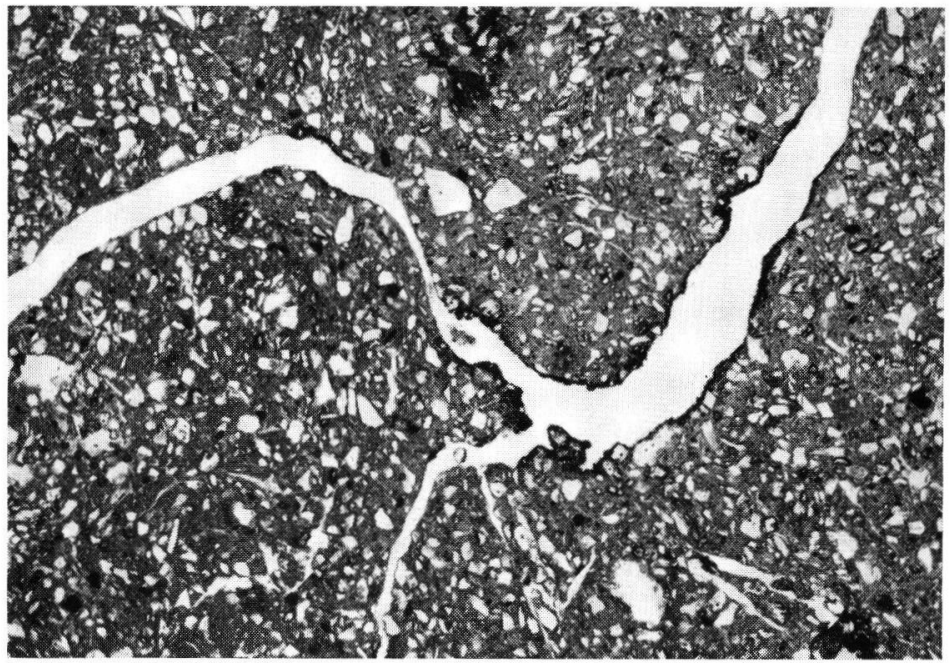

Abb. 11. Fossiler Boden, Probe c: Dichtes, braunlehmartiges Gefüge mit eingeschlossenen Skelettkörnern. Glatte Schwundrisse, teilweise mit Mn-Überzügen. - Techn. Daten wie Abb. 7.

Von den fossilen Böden des Profiles von Kitros können die beiden Horizonte c) und e) gemeinsam besprochen werden, da sie vollkommen gleichartig sind. Nach der Profilbeschreibung könnte man noch annehmen, daß es sich hier um die Tonanreicherungshorizonte von stark ausgeprägten Parabraunerden handelt, deren tonverarmte Oberböden erodiert wurden. Die Dünnschliffuntersuchungen zeigen jedoch, daß die erwarteten Merkmale der Parabraunerde fehlen. In den Bodenhorizonten sind keine Tonbeläge, die auf eine Toneinlagerung schließen lassen, zu erkennen. Es finden sich auch keine Anhaltspunkte dafür, daß solche Tonbeläge früher vorhanden waren. Aus Parabraunerden und Pelosolen sind Aufarbeitungsvorgänge bekannt, die ein vorhandenes Gefüge vollständig überprägen können. Dabei entstehen aber Reliktmerkmale, die den vorausgegangenen Gefügezustand rekonstruieren lassen (Altemüller 1960, 1962). In Kitros zeigen die Bodenhorizonte keine entsprechenden Relikte.

So entsteht zunächst die Aufgabe, nach Möglichkeiten einer Tonanreicherung zu suchen, die nicht auf der Einwaschung aufbauen, für die es in den Horizonten selbst keine sicheren Merkmale gibt. Auch in dem fossilen Boden des Profiles von Hodbina konnten keine Merkmale für eine Toneinwaschung nach Art der Parabraunerden gefunden werden. Durch den ungewöhnlich hohen Dolomitgehalt des Ausgangsmaterials bot sich dort aber der Vergleich zur Bildung der Terra fusca an und man konnte den Tongehalt zunächst einmal als eine Akkumulation aus der Lösungsverwitterung erklären. In Kitros kann mit vergleichbaren Vorgängen nicht gerechnet werden. Selbst wenn man unterstellt, daß der Löß primär höhere Carbonatanteile im Skelett besaß, als heute vorhanden sind, gewinnt man keine befriedigende Basis.

Der Mineralbestand von Kitros deckt aber einen anderen Zusammenhang auf. Alle carbonathaltigen Lösse und Kolluvien enthalten in größerer Menge Muscovit, Biotit, Chlorit und vielleicht andere Phyllosilikate, die in verschiedener Größe und unterschiedlichem Frischezustand im Gefüge verteilt sind. In den Bodenhorizonten fehlen diese Minerale im Skelettanteil fast vollständig und kommen nur noch in feiner Zerteilung mit fließenden Übergängen in die Tonkorngrößen vor. Besonders interessant ist in diesem $\mathrm{Zu}$ sammenhang der schwach entwickelte Bodenhorizont (Probe g). Die Glimmer- und Chlorite sind hier noch sehr zahlreich, sie nehmen aber hinsichtlich ihrer Größe und dem Grad der Zerteilung eine Mittelstellung zwischen den carbonatreichen Ausgangsmaterialien 


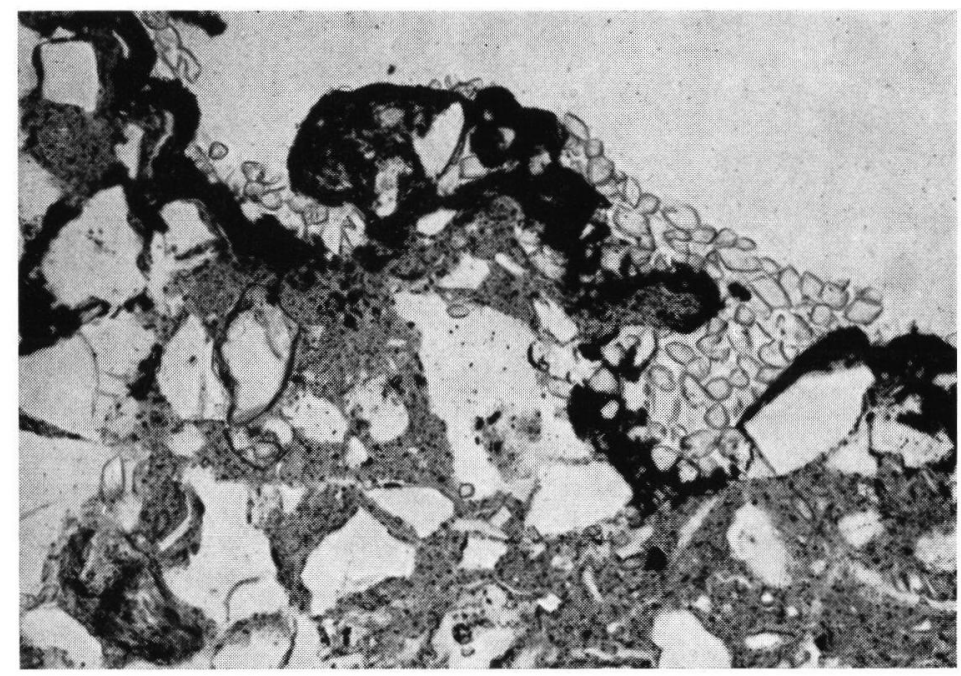

Abb. 12. Fossiler Boden, Probe c: Braunlehmartiges Gefüge bei stärkerer Vergrößerung mit MnÜberzug und einer Ansammlung von Calcitkörnchen. - Techn. Daten wie Abb. 8.

und den Bodenhorizonten ein. Daraus ergibt sich zumindest eine qualitative Beziehung zwischen dem Rückgang des Anteils der Phyllosilikate und der Zunahme des Tonanteils. Irgendwelche anderen Beziehungen zwischen Verwitterungsmerkmalen an Mineralen und der Tonbildung lassen sich aus den Dünnschliffen nicht ableiten. Die beobachteten Lösungserscheinungen an Hornblenden, an Apatit und einigen Plagioklasen vermitteln keine Anhaltspunkte für eine Beziehung zur Tonbildung in solcher Menge.

Obwohl die Bodenhorizonte keine Merkmale einer Toneinwaschung aufweisen, darf die Möglichkeit nicht ausgeschlossen werden, daß Einwaschungen stattgefunden haben. Immerhin sind doch unter den Bodenhorizonten im frischen Löß oder Kolluvium Tonbeläge aus Einwaschungen vorhanden, die aus den Bodenhorizonten stammen. Auch der schwach entwickelte Bodenhorizont g) enthält solche Tonbeläge. Diese Profilabschnitte haben aber alle ein intaktes Leitbahnsystem, das einen Tontransport im wassergesättigten Zustand ermöglicht. Den braunen Bodenhorizonten fehlt dieses. Es muß aber ursprünglich vorhanden gewesen sein, denn es ist für das Ausgangsmaterial kennzeichnend. - Daß die Leitbahnen (Nadelstichporen) heute fehlen, spricht für eine starke Aufarbeitung und $\mathrm{Ge}-$ fügeveränderung. Überraschend ist die Tatsache, daß keine Relikte den früheren Zustand sicher rekonstruieren lassen.

Nach ihrem Gefügebild gleichen die Bodenhorizonte c) und e) einem typischen Braunlehm im Sinne von Kubiëna (1953) ${ }^{2}$ ). Ein wichtiges Merkmal des Braunlehms ist seine starke Neigung zum Dispergieren und Zerfließen. Diese Eigenschaften können sich nur in einem gleichmäßig feuchten Klima entwickeln und erhalten. Sie verlieren sich, wenn periodische Austrocknung oder auch Frostwirkung einsetzt. Dann ist das Bodenmaterial stabilisiert und besser imstande, Trümmer aus Aufarbeitungen über längere Zeit zu erhalten.

Man wird diese Braunlehmeigenschaften auch den Bodenhorizonten von Kitros zuerkennen müssen, wenn eine Erklärung gefunden werden soll für den Mangel an Gefügerelikten aus vorangegangenen Entwicklungsstadien. Es kann also nicht ganz von der

2) Herrn Professor KuBı̈̈NA sei an dieser Stelle für die Durchsicht einiger Schliffpräparate und für wertvolle Hinweise herzlich gedankt. 


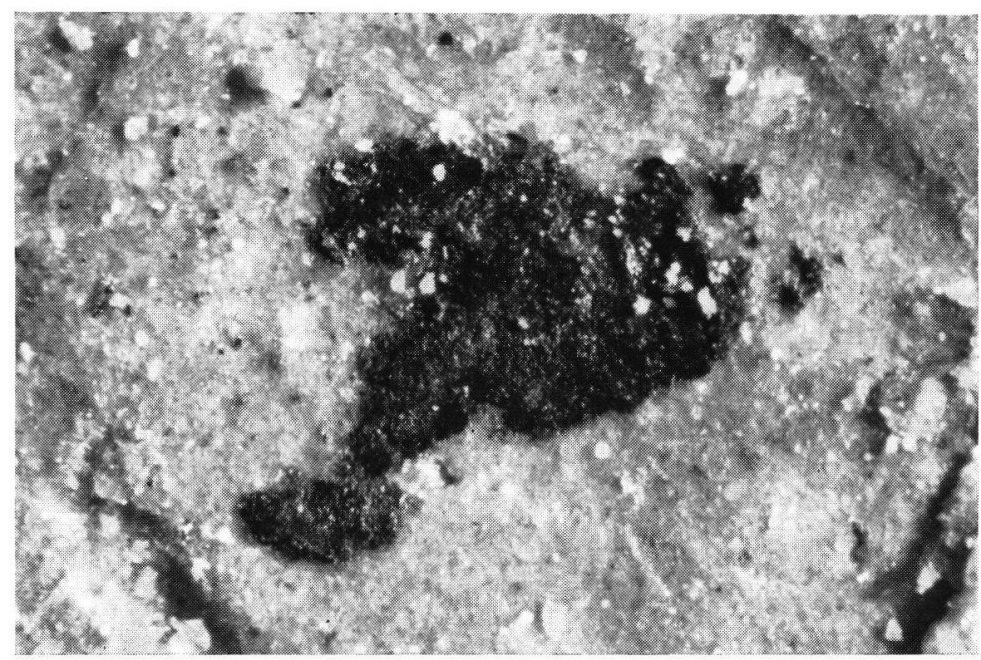

Abb. 13. Fossiler Boden, Probe e: Kluftläche eines frischen, nicht eingebetteten Bodenstückchens im Auflicht. Fleckenartiger Überzug von Mn-Hydroxid. $M=40: 1$. - Objektiv Leitz UO 3,8, Ultropak-Illuminator.

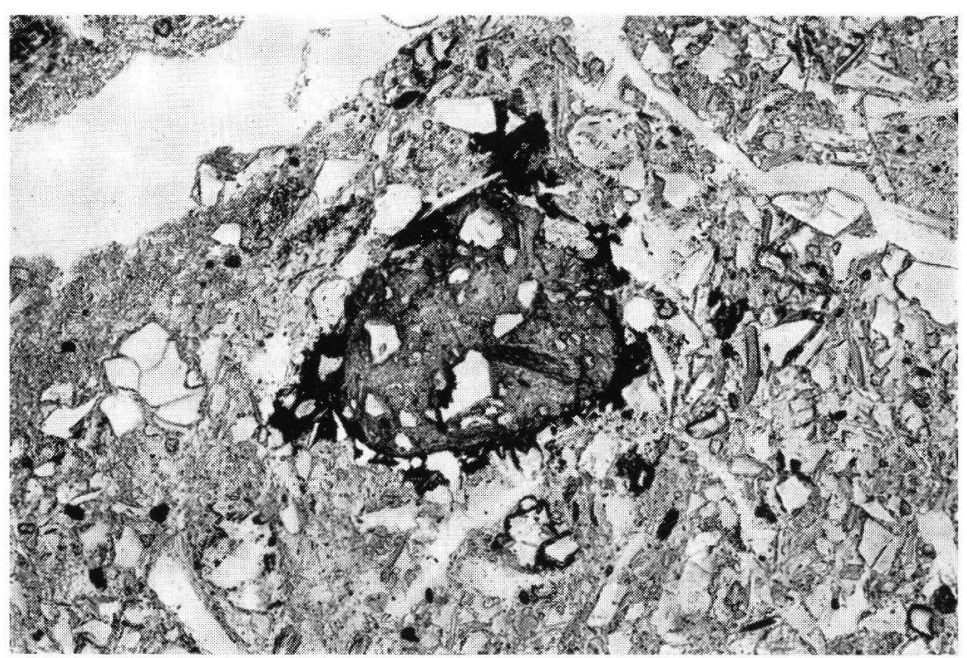

Abb. 14. Kolluvium, Probe h: Geröll eines braunlehmartigen Bodens, umkrustet von Mn-Hydroxid. $\mathrm{M}=100: 1$. - Objektiv Leitz Pl 10/0,25 Hellfeld, Durchlicht.

Hand gewiesen werden, daß auch Toneinwaschungen in die tonreichen Bodenhorizonte erfolgt sind.

Die Ausscheidungen von Manganhydroxiden sind nicht leicht zu bewerten. Grundsätzlich sind sie als Pseudogleymerkmale aufzufassen, denn sie kommen in der Regel zusammen mit Eisenhydroxid-Ausscheidungen vor, sie zeigen aber eine gewisse Unabhängigkeit von den Fe-Ausfällungen. In Kitros ist ihre bevorzugte Ausscheidung an Oberflächen kennzeichnend. Vermutlich sind sie einem relativ späten Entwicklungsstadium zuzuordnen.

Eisenhydroxid-Ausscheidungen sind im gesamten Profil sehr selten. Die am natürlichen Aufschluß beschriebenen Fe-Flecken oder Überzüge erwiesen sich im Dünnschliff als Fe- 
reiche Tonbeläge, die das Eisen in feinstverteilter Form enthalten, wie es für braunlehmartiges Material bezeichnend ist.

Die intensive Bodenbildung in den Horizonten c) und e) ist für die zeitliche Einordnung der Böden von großer Bedeutung. Unter mitteleuropäischen Verhältnissen könnte man solche Böden keinesfalls als interstadiale Bildungen ansehen. Auch für den Mittelmeerraum muß diese Intensität der Bodenbildung in einem Interstadial überraschen. Die Böden müssen eine ganze Folge von Prozessen durchlaufen haben, für die man bisher, auch wenn sie ineinanderwirken, einen nicht geringen Zeitfaktor angesetzt haben würde. Hierzu gehören vor allem

die Entkalkung und Verbraunung,

die Tonbildung durch Verwitterung der Glimmer,

mögliche Tonverlagerungen und -anreicherungen,

die Ausbildung und Steigerung braunlehmartiger Merkmale bei anhaltender Verwitterung und Verdichtung des Gefüges,

der Zusammenbruch des Leitbahnsystems,

Vorgänge der Aufarbeitung und Mischung bis zur Homogenisierung der Grundmasse.

Der schwach entwickelte Boden im Profil von Kitros ist danach lediglich bis zum zweiten Stadium vorgedrungen. Es fehlt jedoch das Merkmal der Verbraunung. Dafür sind die Manganhydroxid-Ausscheidungen dort stärker ausgebildet und deuten auf gewisse Staunässeeinflüsse.

Der unterste Bodenhorizont i) paßt in die aufgestellte Entwicklungsreihe zwanglos hinein. Manches spricht dafür, daß dieser noch eine längere Entwicklung durchlaufen hat. Die Untersuchung von weiterem Material wäre aber zu wünschen.

\section{d. Pollenanaly tische Untersuchungen (H.-J. Beug)}

Die Anreicherung der Pollenkörner (PK) erfolgte mit Hilfe einer Schweretrennung und anschließender Azetolyse. Von jeder Probe wurden dabei $100 \mathrm{~g}$ aufbereitet. Bei den Proben a-d geschah dieses nach dem Verfahren von Frenzel (1964), bei den Proben e-i nach der bei BrunNacker et al. (1969) beschriebenen Methode. Für die Pollenzählungen mußte fast stets der ganze pollenführende Rückstand verwendet werden. Einigermaßen statistisch gesicherte Ergebnisse ließen sich nur bei den Proben e-h erzielen, während in den Proben $a-d$ und i nur wenige PK gefunden wurden (Tab. 1). Die Spektren der pollenreicheren Proben Kitros $\mathrm{e}-\mathrm{f}$ sind in Tabelle 2 zusammengestellt und die Einzelwerte in Prozenten einer Grundsumme berechnet, die aus Baumpollen und Nichtbaumpollenanteilen besteht. Es sei noch darauf hingewiesen, daß in der Probe h nur 18 PK gefunden wurden. Die statistische Sicherung der Einzelwerte ist daher in dieser Probe ganz besonders schlecht.

Bei einer früheren pollenanalytischen Untersuchung südeuropäischer Lösse (BRUNNACKER et al. 1969) konnte gezeigt werden, daß dort der Polleninhalt zum größten Teil von der nacheiszeitlichen Bodendecke her eingedrungen sein mußte. Etwas derartiges kann hier jedoch mit großer Wahrscheinlichkeit ausgeschlossen werden, da in den obersten $3 \mathrm{~m}$ des Profiles - soweit untersucht - nur verschwindend geringe Pollenmengen enthalten sind (Proben a-d).

Auswertbare Unterschiede zwischen den pollenreicheren Spektren $\mathrm{e}-\mathrm{h}$ scheinen nur in den Werten von Pinus, Betula und den klimatisch anspruchsvolleren Laubhölzern (Quercus, Ulmus, Fraxinus, Ostrya, Vitis, cf. Pbillyrea, Corylus, Carpinus und Fagus) vorhanden zu sein. Tabelle 3 führt diese zusammen mit den Anteilen der Baumpollen und Nichtbaumpollen auf. 
Tabelle 1

Pollenkörnerin den Proben a-d und i

$\begin{array}{llll}\text { Kitros a } & \text { Pinus } & 1,5 \mathrm{PK} \\ & \text { Compositae } & 1 & \mathrm{PK} \\ \text { Kitros b } & \text { Pinus } & 1 & \mathrm{PK} \\ \text { Kitros c } & \text { Pinus } & 1 & \mathrm{PK} \\ \text { Kitros d } & \text { Pinus } & 2 & \mathrm{PK} \\ & \text { Alnus } & 1 & \mathrm{PK} \\ & \text { Corylus } & 1 & \mathrm{PK} \\ & \text { Gramineen } & 1 & \mathrm{PK} \\ \text { Kitros i } & \text { Farne } & 1 & \text { Spore } \\ & & 1 & \mathrm{PK}\end{array}$

Tabelle 2

Pollenspektren der Proben e-h

Pinus diploxylon-Typ

Betula

Salix

Ephedra fragilis-Typ

Ericaceae

Ostrya

Vitis

cf. Phillyrea

Quercus

Ulmus

Fraxinus excelsior-Typ

Corylus

Alnus

Carpinus betulus

Fagus

Picea abies

Artemisia

Chenopodiaceae

Compositae

Gramineae

Umbelliferae

Papilionaceae

Ranunculaceae

Polygonum aviculare-'Typ

Plantago lanceolata-Typ

Cyperaceae

Urtica

Cruciferae

Plantago spec.

Onosma-Typ

Rubiaceae

Centaurea jacea-Typ

Unbestimmbare

Summe Baumpollen

Summe Nichtbaumpollen

Gezählte Pollenkörner

Sumpfpflanzen:

Sparganium

Typha
37,5

7,5

1,2

3,8

3,1

0,9

1,2

2,5

2,5

1,2

1,3

2,5

7,5

10,0

2,5

3, 8

11,3

1,2

1,

0,9

1,3

1,3

0,9

0,9

3,5

1,3

1,3

9,0

3,5

9,7

2,6

0,4

1,7

6, 1
0,9

0,4

0,4

0,4

0,4

0,4

3,8
57,4
42,6
80

8,2 h 5,6 27,7 3,3 1,1

1,1

11,1 5,6 1,1

1,1

11,4

16,0

3,4

2, 3

10,2

2,3

5,6

5,6

5, 6

16,6

5,6

11,0

2,3

2,3

47,7

55,6

52,3

88

44,4

18 
Tabelle 3

\begin{tabular}{lrrrr} 
& $\mathrm{e}$ & $\mathrm{f}$ & $\mathrm{g}$ & $\mathrm{h}$ \\
\hline Pinus & 37,5 & 12,7 & 40,9 & 5,6 \\
Betula & 7,5 & 24,8 & 3,3 & 27,7 \\
Klimatisch anspruchsvolle Laubhölzer & 4,9 & 13,6 & 2,2 & 16,7 \\
Summe Baumpollen & 57,4 & 54,6 & 47,7 & 55,6 \\
Summe Nichtbaumpollen & 42,6 & 45,4 & 52,3 & 44,4
\end{tabular}

Niedrige Pinus-Anteile treten demnach zusammen mit hohen Werten der Birke und den anspruchsvollen Laubhölzern auf und umgekehrt. Von h zu e ist dabei ein zweifacher Wechsel von hohen zu niedrigen Anteilen dieser klimatisch anspruchsvollen Laubhölzer zu erkennen. Ein Zusammenhang mit den Änderungen der Nichtbaumpollen-Anteile scheint allerdings nicht zu bestehen. Diese liegen zwischen 42,6 und 52,3\% der Gesamtsumme. Aufgrund dieser Werte kann man im übrigen annehmen, daß es damals bei Kitros keine geschlossenen Wälder gab und höchstens ein spärlicher Baumwuchs möglich war. Auf die regelmäßigen, z. T. sogar häufigen Funde von Chenopodiaceen und Artemisia sei in diesem Zusammenhang besonders hingewiesen.

Es liegt nahe, die Pollenspektren von Kitros mit dem Pollendiagramm aus der DramaEbene zu vergleichen, die etwa $160 \mathrm{~km}$ ONO von Kitros entfernt ist (van DeR Hammen et al. 1965). Für den jüngeren Teil der letzten Kaltzeit geben hier die Autoren sehr hohe Nichtbaumpollen-Anteile (darunter viel Artemisia und Chenopodiaceen) und unter den Baumpollen nur minimale Kiefern-Werte an. Im älteren Teil der Würm-Kaltzeit zeigen die Pollenspektren Nichtbaumpollen-Anteile etwa zwischen 90 und 50\% der Gesamtsumme. Unter den Baumpollen dominiert dabei die Kiefer, und die Laubholz-Anteile (Quercus) liegen etwa zwischen 5 und 10\%. Die Pollenspektren e-h aus Kitros lassen sich also durchaus mit dem vermutlich frühwürmzeitlichen Abschnitt (17,5-24 m Tiefe) des Pollendiagrammes aus dem Drama-Becken vergleichen.

Eine darüber hinausgehende vegetations- und klimageschichtliche Deutung der Pollenspektren ist z. Z. noch sehr schwierig. Über die Pollenflora südeuropäischer Lösse liegen so gut wie keine Erfahrungen vor. Außerdem müßte geklärt werden, ob und wie stark Pollenkörner hier bei Verlagerungsprozessen in den Lössen und Paläoböden mit erfaßt worden sind.

Die höheren Anteile klimatisch relativ anspruchsvoller Laubhölzer in den Spektren $f$ und h scheinen für ein wärmeres und bzw. oder feuchteres Klima als in e und g zu sprechen. Unklar bleibt dann aber die Bedeutung der hohen Pinus-Anteile in e und g (Fernflug?). Eine Klärung dieser offen gebliebenen Fragen kann wohl nur durch weitere Untersuchungen an südeuropäischen Lössen, vielleicht auch durch eine Neubearbeitung des Profiles Kitros mit engerem Probenabstand erfolgen.

\section{e. Zeitliche Einstuf ung (K. Brunnacker)}

Zur Alterseinstufung bieten sich zwei grundsätzlich verschiedene Möglichkeiten an: Entweder wird das Profil durch interglaziale Böden gegliedert, womit die Deckschichten einen erheblichen Bereich des Pleistozäns überspannen würden, oder es handelt sich um interstadiale Bildungen, die sich allerdings auf das Würm beschränken müßten. Trotz der sehr kräftigen Ausprägung der Paläoböden wird die zweite Möglichkeit vorgezogen: Andernfalls müßte in Kitros, wie in anderen Profilen dieses Raumes, eine Abfolge ohne wesentlichere Erosionsdiskordanzen vorliegen, wie sie selbst in solchen Bereichen Mittelund SE-Europas, welche für die Erhaltung reich gegliederter Profile günstiger sind, zu den großen Seltenheiten zählt. - Die Lage zum Talboden und der Aufbau des Profils sprechen, wie angedeutet, für Einstufung in das Jungpleistozän, wobei der Boden i am 
Beginn des Würm stehen würde. - Das bei Estepona in S-Spanien gefundene analoge Profil liegt auf der 10-m-Strandterrasse, die dem letzten Interglazial zugerechnet werden kann (BRUNNACKer \& LožEK 1969). - Interessant ist in diesem Zusammenhang das Verhalten des leichtlöslichen (dithionitlöslichen) Eisens. Dessen Anteil am (HCl-löslichen) Gesamteisen nimmt in Kitros mit dem Alter der begrabenen Böden in ähnlichen Raten ab, wie sie bei entsprechend eingestuften Würm-Paläoböden in Kärlich gefunden wurden (BRUNNACKER 1969).

Letztenendes gibt das Gesetz von der Korrelation der Fazies den Ausschlag: Hinsichtlich der Zahl der zwischengeschalteten Innerwürm-Böden ist ein Vergleich mit den Profilen der feuchteren Randgebiete der Lößverbreitung möglich (BRUNNACKER 1967). Der Ausprägungsgrad dieser Böden wird zudem gegen wärmere Räume hin vor allem dann zunehmen, wenn diese in etwas feuchteren Regionen liegen.

\section{Weitere Vorkommen (K. BRUNNACKER)}

Das Profil von Kitros ist insofern kein Einzelfall, als entsprechende Vorkommen in N-Griechenland bei Xanthi und in Kleinasien östlich von Adapazari entdeckt wurden. Außerdem wurden gleichartige Paläoböden zwischen Gehänge-Spülschutt und Kolluvien nördlich Thessaloniki angetroffen. Möglicherweise gehört das Profil von Kokkinopilos (DaKaris, Higgs \& Hey 1964) ebenfalls dazu.

Damit zeichnet sich in den Tiefländern des nordöstlichen Mittelmeer-Raumes eine eigenständige Löß- und Paläoboden-Provinz ab, die sich durch Zahl und Ausbildung der Innerwürm-Böden deutlich von benachbarten Räumen abhebt. So ist an der Neretva nur ein Boden mit etwas schwächerer Ausprägung im Löß vertreten (vgl. BRUNNACKER u. Mitarbeiter 1969), ähnlich am Vardar in S-Jugoslawien. Im arideren westlichen Anatolien wurde in deutlicher entwickeltem Deluviallöß ebenfalls nur eine „Kalkbraunerde“ gefunden (im Hochland bei Karaman und im Mäander-Tal bei Denizli).

Hingegen liegt in S-Spanien bei Estepona, zwischen Malaga und Gibraltar, ein Kolluvialprofil, das durch Böden unterteilt wird, die denen von Kitros entsprechen. Sie unterscheiden sich makromorphologisch lediglich durch ockerbraune Färbung (BRUNNACKer \& LožEK 1969).

\section{Stellung innerhalb der würmeiszeitlichen Klimazonen (K. BRUNNACKER)}

Bezüglich Lößfazies und örtlichem Profilaufbau wirken Provinz, Humiditätsgrad und Standortgegebenheiten zusammen. Abgesehen von speziellen Lokaleinflüssen lassen sich deshalb die aus einem Profil ablesbaren Gegebenheiten extrapolieren, soweit der Landschaftstyp der gleiche bleibt.

Damit können die für Kitros dargestellten Befunde verallgemeinert werden. Für die Fazies gilt dabei, daß noch typischer Löß vorliegt. Doch muß innerhalb des durch den Löß als solchen angedeuteten Rahmens mit einer gewissen Neigung zu etwas feuchteren Bedingungen gerechnet werden. Dies zeigt sich besonders über die Böden an, wobei deren genaue stratigraphische Position im Würm ohne Belang ist.

Innerhalb der nordmediterranen Länder treten Profile mit einem Aufbau entsprechend Kitros in der Grenzzone des Lösses nach feuchteren Räumen auf. Je humider ein Gebiet ist, desto mehr werden Schwellenwerte wirksam, die für die Einschaltung zusätzlicher Böden und deren Ausprägung verantwortlich sind. Damit steht die durch Kitros repräsentierte Provinz - wie die in Estepona erfaßte - in einer ähnlichen Stellung zu trockenen mediterranen Provinzen, wie die des nördlichen Rheinlandes und des nördlichen Alpenvorlandes zu den trockeneren Bereichen Mitteleuropas (BRunnacker 1967). 
Für die Stadialzeiten darf ein Klima mit weitgehend offener Vegetation unterstellt werden, wie es neuerdings Frenzel (1967) aufgrund eines Pollenprofils in N-Griechenland fordert (van der Hammen, Wijmstra \& van der Molen 1965), und dem die pollenanalytischen Befunde von Kitros nicht entgegen stehen. Dagegen sprechen auch nicht die Faunenfunde von Larissa (Milojčrć, Boessneck, Jung \& Schneider 1965), zumal deren Zeitstellung unklar bleibt.

\section{Pluviale Phasen (K. Brunnacker)}

Die für nordmediterrane Tiefländer skizzierten beiden, etwas humideren Löß- und Paläoboden-Provinzen sind heute durch etwa $16^{\circ} \mathrm{C}$ mittlere Jahrestemperatur bei einem Niederschlagsmittel von 500-600 mm und um 4 Dürremonate gekennzeichnet. Im Würm lag dieser Bereich etwa 400-700 m unter der damaligen Periglazialstufe (i. w. S.), wenn vom Erhaltungszustand der Terrae calcis und (was aber nicht überall ein sinnvolles Kriterium ist) der Untergrenze weitflächiger Gehänge-Spülschutte ausgegangen wird (BRUNNACKER u. Mitarbeiter 1969).

Eine überschlagsmäßige Abschätzung der eiszeitlichen Temperaturdrepression auf Grund der unterschiedlichen Lage der rezenten und würmeiszeitlichen Periglazialstufe bringt, wie an der Neretva und in SE-Spanien eine Absenkung von größenordnungsmäßig $10^{\circ} \mathrm{C}$, also gegen $6^{\circ} \mathrm{C}$ Jahresmittel, im höheren Würm. Doch ist dies eine Frage des nicht sonderlich gut faßbaren Temperaturgradienten. Aber auch nach den bisherigen pollenanalytischen Befunden kommt man zu ähnlichen Vorstellungen; denn die hygrische Situation kann in diesen von zahlreichen großen und kleinen Tälern durchzogenen Tieflandsgebieten nicht die Begrenzung thermophilerer Waldvegetation bestimmen. Man wird deshalb auch in diesen Bereichen auf den Temperaturfaktor zurückgreifen müssen, um das Zurücktreten anspruchsvollerer Bäume zu Gunsten von Steppenelementen erklären zu können. Dafür ist wiederum eine erhebliche Depression Voraussetzung (heutiges Julimittel in Thessaloniki rd. $26^{\circ} \mathrm{C}$ ).

Obiger Wert steht durchaus nicht isoliert, wenn z. B. die Angaben von Messerli (1967) vergleichsweise herangezogen werden. Doch kommt es derzeit gar nicht so sehr auf Interpretationsunterschiede in der Größenordnung einiger Grade an. Wesentlich ist vielmehr, daß man auch für den nordmediterranen Raum eine tiefgreifende eiszeitliche Temperatursenkung zu fordern beginnt. Eine solche könnte wegen der geminderten Verdunstung durchaus Erscheinungen auslösen, die andernfalls durch vermehrte Niederschläge, also durch Pluviale bisheriger Interpretation, erklärt werden mußten (FrEnzel 1967).

Nur soviel sei deswegen dazu bemerkt: Ein „fluviatiles Relief“, das u. a. als Indiz für Pluvialzeiten herangezogen wird, sagt nicht viel; denn auch die weitaus meisten terrestrischen Sedimente, die (eindeutig) ariden Zeiten der Erdgeschichte zugesprochen werden, sind fluviatiler Herkunft (i. w. S.). Sowohl in deren Abtrags- wie Akkumulationsräumen muß damit ein derartiges fluviatiles Relief dominiert haben.

Die interstadialen Phasen der periglazialen Gebiete werden gegen Süden zu pluvialen Phasen, deren Hintergründe vorerst offen bleiben. Gemäß der Abhängigkeit vom jeweiligen entscheidenden "Schwellenwert" wird jedoch eine gebietsweise unterschiedlich große Zahl humiderer Abschnitte über die Böden faßbar. Denkbar ist ferner, daß der Beginn und das Ende der interstadial-pluvialen Bodenbildungen in wärmeren Bereichen früher eingesetzt und später geendet hat als im periglazialen Raum. Doch würde sich damit am Grundsätzlichen nichts ändern. Soweit Hinweise vorliegen, deuten die Interstadialböden insgesamt weniger auf stärkere Erwärmung als vielmehr auf etwas feuchtere Bedingungen hin. Unter solchen Aspekten sind wohl auch die genetisch noch recht problematischen „Mediterranböden" zu bewerten. Sie fügen sich in folgende Catena ein (Interstadiale des höheren Würm, z. B. Stillfried B): 
rel. feucht:

Naßboden (Tundragley), insb. nördlich der Alpen

„Braunerde“ (i. w. S.),

z. B. bei Zandar

Mediterranboden, Typ Hodbina, im Neretva-Tal

Mediterranboden, Typ Kitros, zwischen Kitros und Adapazari und bei Estepona rel. trocken:

Brauner Boden (brauner Verwitterungshorizont u. dgl.), in Trockengebieten nördlich der Alpen

Tschernosem im pannonischen Raum

„Kalkbraunerde“, in Anatolien und

SE-Spanien

In Kitros werden gemäß der gebrachten stratigraphischen Interpretation eine kräftige pluviale Phase am Beginn des Würm (i) und eine weitere, schwächere, im Stillfried B (c) wirksam. Dazwischen sind noch zwei pluviale Phasen vorhanden (g, e), deren Wertigkeit anscheinend nach trockeneren Räumen hin wieder unter einen Schwellenwert zurück sinkt. So scheint, ähnlich dem trockenen SE-Spanien (BRUNNACKER \& LOŽEK 1969) der Löß in Nordafrika nur durch einen Kalkausscheidungshorizont (im höheren Würm) unterteilt zu sein (SCHWEgler 1944). Dies spricht keinesfalls gegen eine pluviale Hauptphase wie gegen allgemein etwas feuchtere Bedingungen am Beginn des Würm, zumal in den Sedimenten der Höhle der Crvena Stijena vom Süden kommendes lößartiges Material (wie in der Romanelli-Grotte) auftritt, das aus einem zuvor (etwa am Beginn des Würm) kräftiger chemisch verwittertem Substrat abzustammen scheint (BRUNNACKER 1967).

\section{Schriftum}

AltemüLleR, H.-J.: Mikromorphologische Untersuchungen an einigen Gipskeuperböden im Raum Iphofen/Franken. - Bayer. Landw. Jb., 37, 4, 70—85, München 1960.

- : Mikromorphologische Untersuchungen an Bodenprofilen des mittleren fränkischen Jura. Bayer. Landw. Jb., 39, 1, 98-109, München 1962.

Brunnacker, K.: Die Sedimente der Crvena Stijena. - Glasnik, N. S., 21/22, 31-65, Sarajevo 1967.

- : Die regionale Stellung der niederrheinischen Lößprovinz. - Sonderveröff. Geolog. Inst. Univ. Köln, 13, 55-63, Köln 1967.

- : Ein Löß-Vorkommen bei Kitros/Südmakedonien. - Akademia Athenon, Athen 1968 [in Druck].

— : Kriterien zur relativen Datierung quartärer Paläoböden? - Z. Geomorphologie, 1969 [in Druck].

Brunnacker, K., Basler, Dj., Ložek, V., Beug, H.-J. \& Altemüller, H.-J.: Zur Kenntnis der Lösse im Neretva-Tal. - N. Jb. Geol. Paläontol., Abh. 132, 127-154, Stuttgart 1969.

Brunnacker, K. \& LožEk, V.: Löß-Vorkommen in Südostspanien. - Z. Geomorphologie, Stuttgart 1969.

Dakaris, S. I., Higgs, E. S. \& Hey, W. R.: The Climate, Environment and Industries of Stone Age Greece: Part I. - Proc. Prehist. Soc., 30, 199-244, London 1964.

Fotakiewa, Em. \& Minkow, M.: Der Löß in Bulgarien. - Eiszeitalter u. Gegenwart, 17, 87-96, OOhringen 1966.

Frenzel, B.: Die Klimaschwankungen des Eiszeitalters. — Braunschweig 1967.

Hammen, Th. v. D., Wijmstra, T. A. Molen, W. H. v. D.: Palynological Study of a very thick Peat Section in Greece, and the Würm-Glacial Vegetation in the Mediterranean Region. Geol. en Mijnbouw, 44, 37-39, 's-Gravenhage 1965.

KubIËNA, W. L.: Bestimmungsbuch und Systematik der Böden Europas. - 392 S., Stuttgart 1953.

Marković-Marjanović, J.: Le loess en Yougoslavie. - Rep. VI. Intern. Congr. Quaternary, Warsaw 1961, IV, 551-570, Lodz 1964. 
Messerli, B.: Die eiszeitliche und die gegenwärtige Vergletscherung im Mittelmeerraum. - Geographica Helvetica, 22, 105-228, Bern 1967.

Milojćić, V., Boessneck, J., Jung, D. \& Schneider, H.: Paläolithikum um Larissa in Thessalien. - Beitr. z. ur- u. frühgesch. Archäologie des Mittelmeer-Kulturraumes, 1, Bonn 1965.

Schwegler, E.: Bemerkungen zum Vorkommen von Löß im libyschen und tunesischen Gebiet. N. Jb. Min. etc., Abt. B., Mh. 1944, 10-17, Stuttgart 1944.

Schwertmann, U.: Die Bildung von Goethit und Hämatit in Böden und Sedimenten. - Proc. Intern. Clay Conference, 1, 159-165, Jerusalem 1966.

Manuskr. eingeg. 20. 2. 1969.

Anschriften der Verf.:

Dr. H.-J. Altemüller, Institut für Bodenbearbeitung der Forschungsanstalt für Landwirtschaft, 33 Braunschweig, Bundesallee 50.

Doz. Dr. H.-J. Beug, Botanisches Institut der Universität, 7 Stuttgart-Hohenheim.

Prof. Dr. K. Brunnacker, Geologisches Institut der Universität, 5 Köln, Zülpicher Straße 49. 